\section{MS39-O3 Simulations of single-pulse Laue diffraction from proteins with radiation from synchrotron and XFEL sources}

\author{
Victoria Kabanova ${ }^{1}$, Marius Schmidt ${ }^{2}$, Friedrich Schotte ${ }^{3}$, Marco \\ Cammarata $^{4}$, Michael Wulff
}

1. European Synchrotron, 71, avenue des Martyrs, 38000 Grenoble, France

2. University of Wisconsin-Milwaukee, Physics Department, 3135 North Maryland Avenue Milwaukee, Wi 53211, USA.

3. National Institutes of Health, Lab. of Chemical Physics, NIDDK, Bethesda, Maryland 20892-0520, USA

4. Department of Physics, University of Rennes 1, IPR, UMR 6251, 35042 Rennes, France.

email: victoria.kabanova@esrf.fr

Laue diffraction is the method of choice for measuring ultrafast structural changes in proteins initiated by light. The advantage is that Laue exploits the high intensity of the white beam and that the diffracted intensities are fully recorded with a stationary crystal. In practice, the precision is limited by the signal to noise ratio which depends on many parameters: crystal quality, crystal size, pulse intensity, the fraction of the sample volume activated by the light pulse etc. The time resolution is 100 ps for synchrotrons (SRs) and 100 fs for XFELs. The pulse intensities vary from $1 \times 10^{9}$ to $1 \times 10^{10}$ for SRs and to $1 \times 10^{11}$ to $1 \times 10^{12}$ for XFELs. Finally, the bandwidths are $0.1 \%$ for XFELs and $2.5-3.5 \%$ for SRs.

With the extreme intensity of the XFEL pulse, high quality Laue data can be obtained from micron size crystals by serial crystallography [1]. The number of reflections is limited by the narrow bandwidth; in practice there are 50-100 reflections per image. The crystal is destroyed but the diffraction is generated before this happens. The SR pulse requires larger crystals, 10-300 $\mathrm{mm}$, to get sufficient diffracted intensity [2]. SR Laue with micro crystals is prone to radiation damage: if a $10^{10}$ ph pulse at $15 \mathrm{keV}$ is focused to $\varnothing 10 \mathrm{~mm}$ on a $10 \times 10 \times 10$ $\mathrm{mm}^{3}$ crystal of myoglobin crystal, the temperature rises by $12.4 \mathrm{~K}$ which is often acceptable. For a $5 \times 5 \times 5 \mathrm{~mm}^{3}$ and $\varnothing 5 \mathrm{~mm}$ focus, the temperature rise is $49.5 \mathrm{~K}$ ! The lattice is damaged during the $100 \mathrm{ps}$ exposure.

Motivated by the enormous gain in brilliance with the Extremely Bright Lattice (EBS) at the ESRF (Sep 2020), we are studying the gains from the increased photon density and having a tuneable focus from compound refractive lenses. What is the resolution as a function of crystal size at the EBS? To shed light on this question, we have calculated Laue patterns and intensities including the diffuse background from myoglobin crystals of different size. The simulations use the spectrum from a $\mathrm{U} 14$ cryo-undulator $\left(\mathrm{E}_{\mathrm{f}}=15 \mathrm{keV}, 10^{10} \mathrm{ph} / \mathrm{pulse}, 2.3 \%\right.$ bw, spot $\varnothing 5-100 \mathrm{~mm})$. The Laue patterns are simulated for the Rayonix HS170 detector used at ID09 which has a pixel size of $44.3 \mathrm{~mm}$ and point-spread function of 100 $\mathrm{mm}$. The resolution as a function of crystal size and pulse intensity will be presented.

[1] T.R. Barends et al., Science, 350, 445-450, 2015

[2] F. Schotte et al., Science, 300, 1944-1947, 2003.

Keywords: Laue diffraction, single-pulse experiment, radiation damage, undulator radiation

\section{MS39-04 Time-resolved photocrystallography on a laboratory diffractometer on the millisecond time scale}

Dominik Schaniel $^{1}$, Nicolas Casaretto ${ }^{1}$, Sébastien Pillet ${ }^{1}$, Bertrand Fournier $^{1}$, Pascal Parois ${ }^{1}$, Paul Allé ${ }^{1}$, Emmanuel Wenger

\section{Université de Lorraine, CRM2, Vandoeuvre-les-Nancy, France}

email: dominik.schaniel@univ-lorraine.fr

We have developed a laboratory x-ray diffraction instrument allowing for time-resolved photocrystallography by coupling a ns Nd-YAG pulsed LASER to a fast $\mathrm{x}$-ray pixel detector (XPAD). The very first convincing results have been obtained on time-resolved photoswitching of nitrosyl complexes with this setup. In metal-nitrosyl complexes like sodiumnitroprusside ( $\left.\mathrm{SNP}, \quad \mathrm{Na}_{2}\left[\mathrm{Fe}(\mathrm{CN})_{5} \mathrm{NO}\right] 2 \mathrm{H}_{2} \mathrm{O}\right)$ photoinduced nitrosyl linkage isomers can be generated by irradiation with light in the visible spectral range. Their lifetime varies from a few nanoseconds to several tens of milliseconds at room temperature and increases with decreasing temperature. On the example of the prototype substance SNP we show that the variation of the intensities of Bragg reflections can be monitored with a time-resolution of $2 \mathrm{~ms}$ after pulsed LASER excitation. The photogeneration of the side-on bonded nitrosyl isomer (MSII) and its relaxation back to the ground state could be tracked in this manner at a temperature of 180 $\mathrm{K}$, where the lifetime of SII is of the order of $100 \mathrm{~ms}$. Further examples include ruthenium nitrosyl complexes with lifetimes of the photogenerated linkage isomers of a few miliseconds at room temperature. These proof-of-principle experiments open the way to other applications of this time-resolved photocrystallographic setup.

Keywords: photocrystallography 